\title{
Metastasized murine oral squamous cell carcinoma cells induce intratumoral polymorphonuclear myeloid derived suppressor cells
}

\author{
SHIGEKI SUMI $^{1,2}$, NAOKI UMEMURA ${ }^{2}$, EIJI TAKAYAMA ${ }^{2}$, EMIKA OHKOSHI $^{3}$, \\ MAKOTO ADACHI $^{1}$, MASAKO MIZUNO-KAMIYA ${ }^{4}$, TOSHIHIRO INAGAKI ${ }^{5}$, HARUMI KAWAKI ${ }^{2}$, \\ SHINICHIRO SUMITOMO ${ }^{1}$ and NOBUO KONDOH ${ }^{2}$
}

\begin{abstract}
Departments of ${ }^{1}$ Oral and Maxillofacial Surgery, and ${ }^{2}$ Oral Biochemistry, Asahi University School of Dentistry, Gifu 501-0296; ${ }^{3}$ Department of Natural and Medicinal Chemistry, Faculty of Pharmaceutical Sciences Aomori University, Aomori 030-0943; ${ }^{4}$ Chemistry Laboratory Department of Management and Information Studies, Asahi University School of Business Administration, Gifu 501-0296; ${ }^{5}$ Department of Oral and Maxillofacial Surgery, Division of Reparative and Regenerative Medicine, Institute of Medical Science, Mie University Graduate School of Medicine, Tsu 514-8507, Japan
\end{abstract}

Received October 5, 2016; Accepted March 20, 2017

DOI: $10.3892 / o r .2017 .5575$

\begin{abstract}
Myeloid derived suppressor cells (MDSCs) localize to hematopoietic organs and peripheral blood during inflammation or tumor tissues and lymph nodes in the presence of a tumor. However, whether there are differences in MDSCs found in the primary tumor and metastases is unknown. In the present study, we established a cell line of metastasized tumor cells to a lymph node, L5-11, which were derived from the Sq-1979 mouse buccal mucosa squamous cell carcinoma cell line. We then analyzed tumor immunogenicity, especially with regard to MDSCs, to clarify the differences between the primary tumor and metastases, using an isogenic heterotopic tumor transplantation model. Our data showed that the population of intratumoral MDSCs, especially polymorphonuclear MDSCs in the lymph node metastasis model were significantly increased compared with syngeneic grafts from the primary cell line Sq-1979 after 21 days. Furthermore, we identified that the lymph node metastasis cell line had increased expression of genes that promote the expansion of MDSCs, tumor growth and metastasis. Hence, these data suggest that tumor immunosuppression can occur via activation of MDSCs. However, further examination is required to clarify whether all or a subset of these factors from the lymph node metastasis tumor cells are required to induce intratumoral MDSCs.
\end{abstract}

Correspondence to: Dr Naoki Umemura, Department of Oral Biochemistry, Asahi University School of Dentistry, 1851 Hozumi, Mizuho, Gifu 501-0296, Japan

E-mail: umemura@dent.asahi-u.ac.jp

Key words: immunosuppression, lymphatic metastasis, CD11b antigen, Ly6C antigen, mouse, interleukin-6

\section{Introduction}

The prognosis of oral squamous cell carcinoma (OSCC), which includes head and neck squamous cell carcinoma, is greatly influenced by metastases, such as to the lymph node. The 5-year survival rate of patients without lymph node metastases is between 63 and $86 \%$, while the 5 -year survival rate of patients with lymph node metastases is between 20 and $36 \%(1,2)$. Although traditional thinking suggests the relationship between lymph node metastasis and prognosis is a consequence of the malignancy (3), metastasizing capacity (4) or acquisition of resistance to chemotherapy (5) of tumor cells, the involvement of the immune system in the metastatic process remains poorly explored. To better understand this relationship, we analyzed tumor immunological properties between primary tumor cells and cells from a lymph node metastasis using the L5-11 cell line that we established. The L5-11 cell line was derived from a lymph node metastasis from the murine OSCC cell line Sq-1979. Mice transplanted with the L5-11 cells had larger spleens and tumors than those transplanted with Sq-1979. Therefore, we hypothesized that a tumor immunosuppressive mechanism was acquired by the lymph node metastasis cell line L5-11.

Recently, the presence of abnormally differentiated bone marrow-derived cells has been appreciated as a major immunological characteristic of cancer (6). Many studies have also demonstrated that myeloid derived suppressor cells (MDSCs) downregulate the immune response to tumors, infectious diseases and inflammation $(7,8)$. MDSCs are subdivided into two groups by phenotype: i) monocytic MDSC (M-MDSC), which assume the form and phenotypes of immature mononuclear cells and have the cell surface markers $\mathrm{CD}_{11 b^{+}}, \mathrm{Ly}_{6 \mathrm{C}} \mathrm{C}^{\mathrm{hi}}$ and $\mathrm{Ly}^{-} \mathrm{G}^{-}$; and ii) polymorphonuclear MDSC (PMN-MDSC), which assume the form and phenotype similar to immature polymorphonuclear cells and have the cell surface markers $\mathrm{CD}_{11 b^{+}}$, Ly6C $\mathrm{C}^{\mathrm{lo}}$ and $\mathrm{Ly} 6 \mathrm{G}^{+}(9)$. MDSCs inhibit T cell function (10), induce regulatory $\mathrm{T}$ cells (11) and support tumor growth and metastasis (12), all of which serve a pro-tumor 
function. MDSC from peripheral lymphoid organs suppress antigen-specific $\mathrm{CD}^{+} \mathrm{T}$ cells, in contrast, MDSC from a tumor microenvironment suppress both antigen-specific and non-specific T cell activity (13). However, the activity of MDSCs from a metastasized tumor have not been studied, nor have basic comparisons of MDSCs derived from primary and metastasized tumors. Moreover, there are few studies regarding the role of MDSCs in OSCC. Therefore, we aimed to clarify the characteristics of MDSCs derived from primary tumors and metastases, and our data suggest that the poor prognosis associated with metastasis in OSCC involves tumor immunosuppression.

\section{Materials and methods}

Mice. Male $\mathrm{C} 3 \mathrm{H} / \mathrm{HeN}$ mice, 6-8 weeks old, were purchased from Chubu Kagaku Shizai, Co., Ltd. (Nagoya, Japan). The mice were maintained ad libitum on Oriental MF solid chow (Oriental Yeast, Co., Ltd. Tokyo, Japan). The present study was approved by the Animal Ethics Committee of Asahi University (no. 14-018 and 16-017).

Cell culture and establishment of a metastasized cell line. The C3H murine OSCC cell line Sq-1979 was obtained from the RIKEN BioResource Center (Ibaraki, Japan). Tumor cells were cultured in minimum essential medium (MEM; Wako Pure Chemical Industries, Ltd., Osaka, Japan) containing $10 \%$ fetal bovine serum (FBS; Biowest, Nuaille, France) and antibiotics (100 U/ml penicillin and $100 \mu \mathrm{g} / \mathrm{ml}$ streptomycin) at $37^{\circ} \mathrm{C}$ in a humidified atmosphere containing $5 \% \mathrm{CO}_{2}$.

Sq-1979 cells $\left(1 \times 10^{7}\right)$ were suspended in $0.1 \mathrm{ml}$ of phosphate-buffered saline (PBS), then subcutaneously inoculated in the posterior neck area of mice. After 3 months, metastasized regional lymph nodes were dissected into MEM supplemented with $10 \% \mathrm{FBS}$ and minced to isolate attached cells. Then, the metastasized sub-clone, L5-11 was isolated by the serial limiting dilution method. Cell images were taken using a Nikon Eclipse Ti inverted microscope system (Nikon, Tokyo, Japan).

Evaluation of cell growth using the MTT assay. Sq-1979 or L5-11 cells were seeded into 96-well plates at a density of $1 \times 10^{3}$ cells/well and allowed to adhere for $24 \mathrm{~h}$. Cell viability was assessed daily by addition of $5 \mu \mathrm{l}$ of 3-(4,5-dimethylthiazol-2-yl)-2,5-diphenyltetrazolium bromide (MTT) using a Cell Proliferation kit I (Roche Diagnostics GmbH, Mannheim, Germany) according to the manufacturer's instructions. The number of viable cells was assessed by measuring the absorbance of the produced formazan crystals at $595 \mathrm{~nm}$ with a Tecan SpectraFluor Plus XFluor4 software (Tecan Japan, Co., Ltd., Kawasaki, Japan). The measurement was performed daily for 4 days. Cell growth was calculated relative to the value on the first day, which was set at $100 \%$.

In vivo tumor growth analysis. Sq-1979 or L5-11 cells $\left(1 \times 10^{6}\right)$ were resuspended in $0.1 \mathrm{ml}$ PBS and subcutaneously injected into the lateroabdominal area of male $\mathrm{C} 3 \mathrm{H} / \mathrm{HeN}$ mice. Tumor size was observed every 3 days; the tumor volume was calculated using the formula: $a \mathrm{x} \mathrm{b}^{2} / 2$ where ' $\mathrm{a}$ ' is the length and ' $b$ ' is the width of tumor diameter. Mice were sacrificed after
30 days. There were no significant differences in body weight of the mice.

Preparation of tumor infiltrating cells and spleen cells. Mice were sacrificed 21 days after tumor implantation for analysis. Tumor tissue was finely chopped, incubated with the enzyme mix from the tumor dissociation kit (MACS Miltenyi Biotec; Miltenyi Biotec GmbH, Berdish-Gladbach, Germany) according to the manufacturer's instruction, and then the resuspended-sample was applied to a MACS SmartStrainers (70 $\mu \mathrm{m})$ placed on a 15-ml conical. Red blood cells in the sample were removed using a red blood cell lysis solution (MACS Miltenyi Biotec). For total spleen cell isolation, red blood cells were removed using the same procedures, and the cells were applied to a MACS SmartStrainers $(70 \mu \mathrm{m})$ placed on a $15-\mathrm{ml}$ conical. After collection, total cell numbers were counted with a counting chamber.

Flow cytometry. Fc receptors in the sample were blocked by anti-mouse CD16/32 (eBioscience, Santa Clara, CA, USA) using $1 \mu \mathrm{g}$ per million cells for $10 \mathrm{~min}$ on ice. Then, cells were labeled with Mouse MDSC Flow Cocktail 2 with isotype control, which contained PE anti-mouse CD11b (clone M1/70), FITC anti-mouse Ly-6G (clone 1A8), and APC anti-mouse Ly6C (clone HK1.4), or FITC anti-mouse CD8a clone:53-6.7, PE anti-mouse CD4 clone:GK1.5 (all from Sony Biotechnology, Inc., San Jose, CA, USA), and FITC mouse IgG2 and PE-mouse IgG2b (BD Biosciences, San Diego, CA, USA) for $30 \mathrm{~min}$ in the dark. Cells were then washed and resuspended in FACS buffer (PBS with 0.5\% BSA). Data acquisition and analysis were performed using EC800 flow cytometry analyzer with EC800 analysis software (Sony Biotechnology Inc.).

Real-time PCR. Total RNA was purified using the RNeasy Mini kit (Qiagen, Heiden, Germany), and $600 \mathrm{ng}$ total RNA was used for reverse transcription with iScript ${ }^{\mathrm{TM}}$ Advanced cDNA Synthesis kit (Mediatech, Herndon, VA, USA). For real-time PCR analysis, $1 \mu \mathrm{l}$ cDNA samples diluted 20x, $1 \mu \mathrm{l}$ each of the upper and lower primer (Final $500 \mathrm{nM}$ ), $7 \mu \mathrm{l}$ nuclease-free water, and $10 \mu \mathrm{l}$ SsoAdvanced SYBR-Green Supermix (Bio-Rad Laboratoties) were used. The following primers were designed by Primer Express software (Biosystems, Waltham, MA, USA): VEGFA forward, 5'-GCTGTGCAGG CTGTAACGAT-3' and reverse, 5'-GGTCTGCATTCACATCT GCTGTGC-3'; VEGFR1 forward, 5'-ATCTATAAGGCAGC GGATTGACCG-3' and reverse, 5'-CACGGAGGTGTTGAA AGACTGGA-3'; Npn1 forward, 5'-AATCGAAAACCCAG GGTACCTCAC-3' and reverse, 5'-GCAGTCTCTGTCCTCC AAATGGAA-3'; Npn2 forward, 5'-ACTGTACCTTCACC ATCCTGGCC-3' and reverse, 5'-GGTCCAACATGTGG AATGCCATC-3'; Sema3a forward, 5'-AGATGCTCCATT CCAGTTTGTTCAC-3' and reverse, 5'-ACATAAGCCACCG CATCACTTGTA-3'; Sema3b forward, 5'-GGATGCATGT CTCTGAGCTCCG-3' and reverse, 5'-GGCCCAGCCATAA CTCATTTGTC-3'; Sema3f forward, 5'-ATGGCTGATAT CCGCATGGTCTT-3' and reverse, 5'-CCTTAGTGGACTT CATCGAGGGC-3'; S100A9 forward, 5'-CATCATGGAGG ACCTGGACACAA-3' and reverse, 5'-GCAGCTTCTCA TGACAGGCAAAGA-3'; IL-6 forward, 5'-CCTACCCCA ATTTCCAATGCTCTC-3' and reverse, 5'-GCATAACGCA 

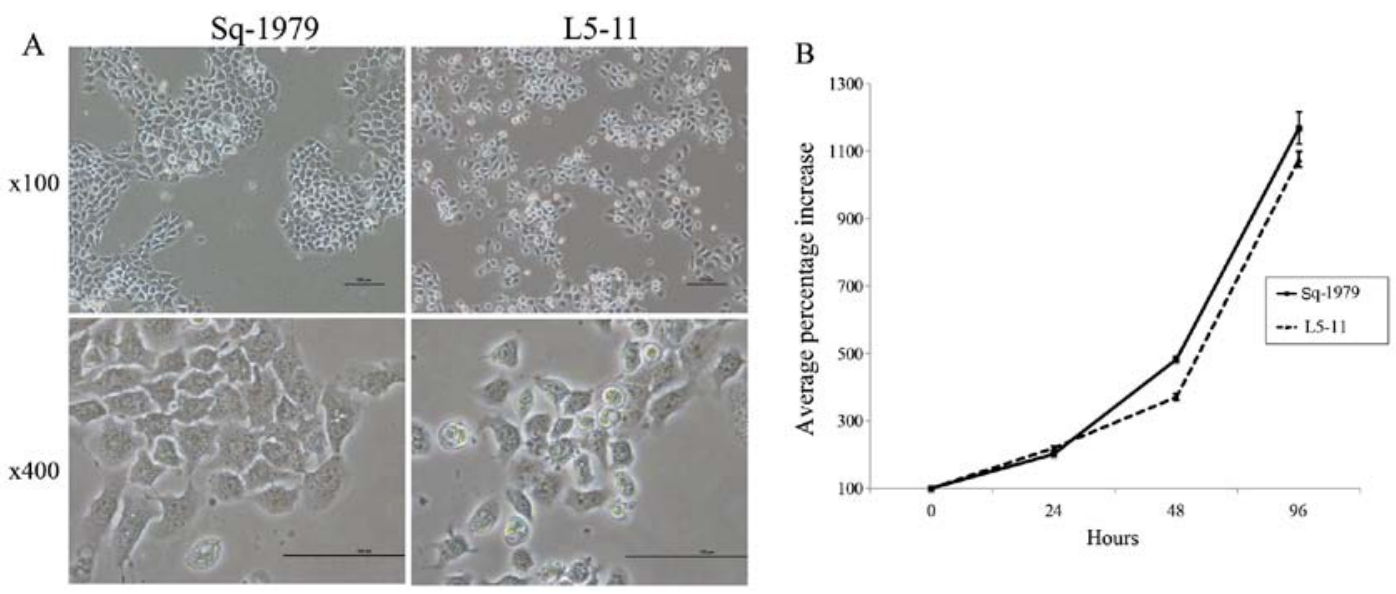

Figure 1. In vitro characterization of Sq-1979 and L5-11 cells. (A) Sq-1979 cells (left panel) and L5-11 cells (right panel) were imaged at x100 magnification (top panel) and x400 magnification (bottom panel). The scale bar is $100 \mu \mathrm{m}$. (B) Sq-1979 and L5-11 were seeded into 96-well plates at a density of 1x10 cells/well and allowed to adhere for $24 \mathrm{~h}$. Cell viability was assessed on a daily basis by the MTT assay.
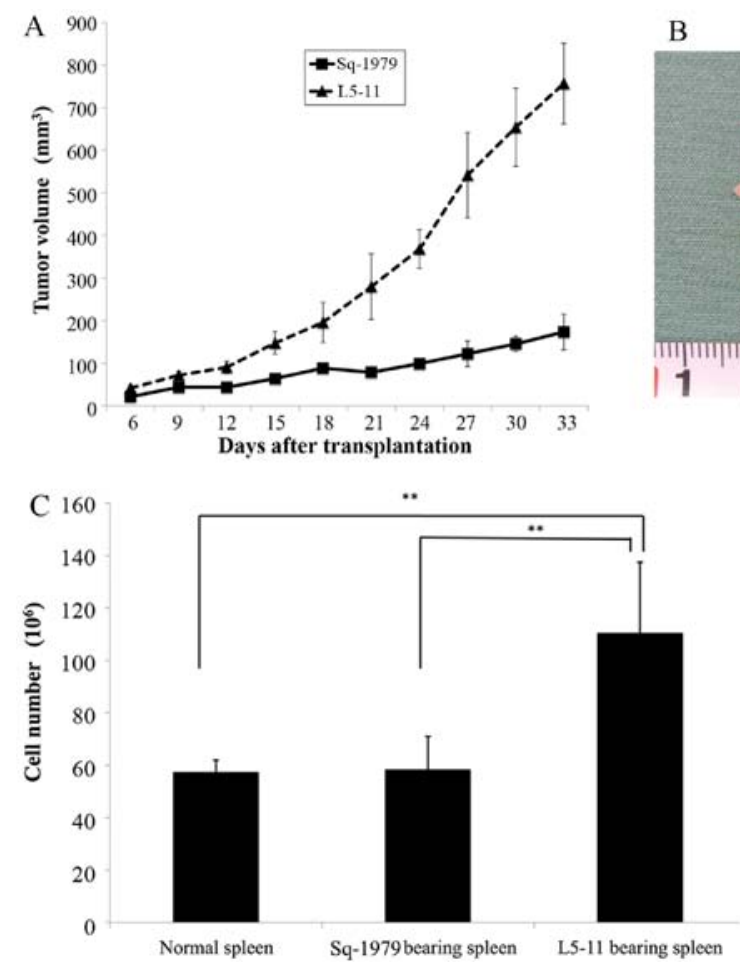
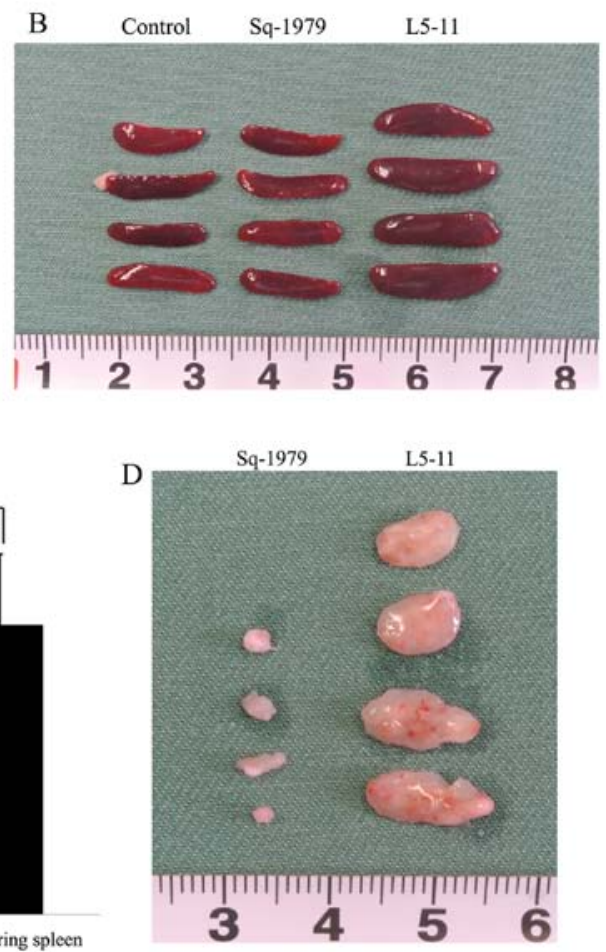

Figure 2. In vivo tumor growth. (A) Tumor growth curves for Sq-1979 and L5-11 xenografts. Mice were inoculated by subcutaneous injection on day 0 with $1 \times 10^{6} \mathrm{Sq}-1979$ or L5-11 cells. The y-axis shows tumor growth, and the x-axis shows the days after the transplantation. Tumor volume was measured every 3 days. (B-D) After 21 days of syngeneic grafts growth spleens and tumors were removed from recipient animals (n=13). (B) Representative spleens are shown from negative control mice (left panel), Sq-1979-transplanted mice (middle panel) and L5-11-transplanted mice (right panel). (C) Bar graph indicates the total splenic cell numbers. (D) Representative tumors are shown from Sq-1979 syngeneic grafts (left panel) and L5-11 syngeneic grafts (right panel). All quantitative data are represented as mean $\pm \mathrm{SD}$ of three independent experiments. ${ }^{*} \mathrm{P}<0.05,{ }^{* * *} \mathrm{P}<0.01$.

CTAGGTTTGCCG-3'; and HIF1 $\alpha$ forward, 5'-TGGATTT TGGCAGCGATGACAC-3' and reverse, 5'-AGTGGCTTTG GAGTTTCCGATGA-3'. The reaction conditions consisted of one 5 -min cycle at $95^{\circ} \mathrm{C}$, followed by 45 cycles at $95^{\circ} \mathrm{C}$ for $10 \mathrm{sec}$, and $72^{\circ} \mathrm{C}$ for $10 \mathrm{sec}$. The reaction and absolute quantitation analysis were performed with a Thermal Cycler Dice ${ }^{\circledR}$ Real-Time System TP800 (Takara Bio, Inc., Shiga, Japan).

Statistical analysis. All quantitative data are presented as the mean \pm SD. Data were evaluated using one-way analysis of variance followed by Tukey-Kramer test for Figs. 2C, 3B and C, 4B and D. Unpaired two-tailed Student's t-test was performed for the two-group comparisons in Figs. $3 \mathrm{E}$ and $\mathrm{F}$ and $4 \mathrm{E}$ and $\mathrm{F} . \mathrm{P}<0.05$ was considered statistically significant.

\section{Results}

Characterization of the murine lymph node metastasis cell line derived from OSCC. After establishing the L5-11 murine lymph node metastasis cell line, derived from the murine 

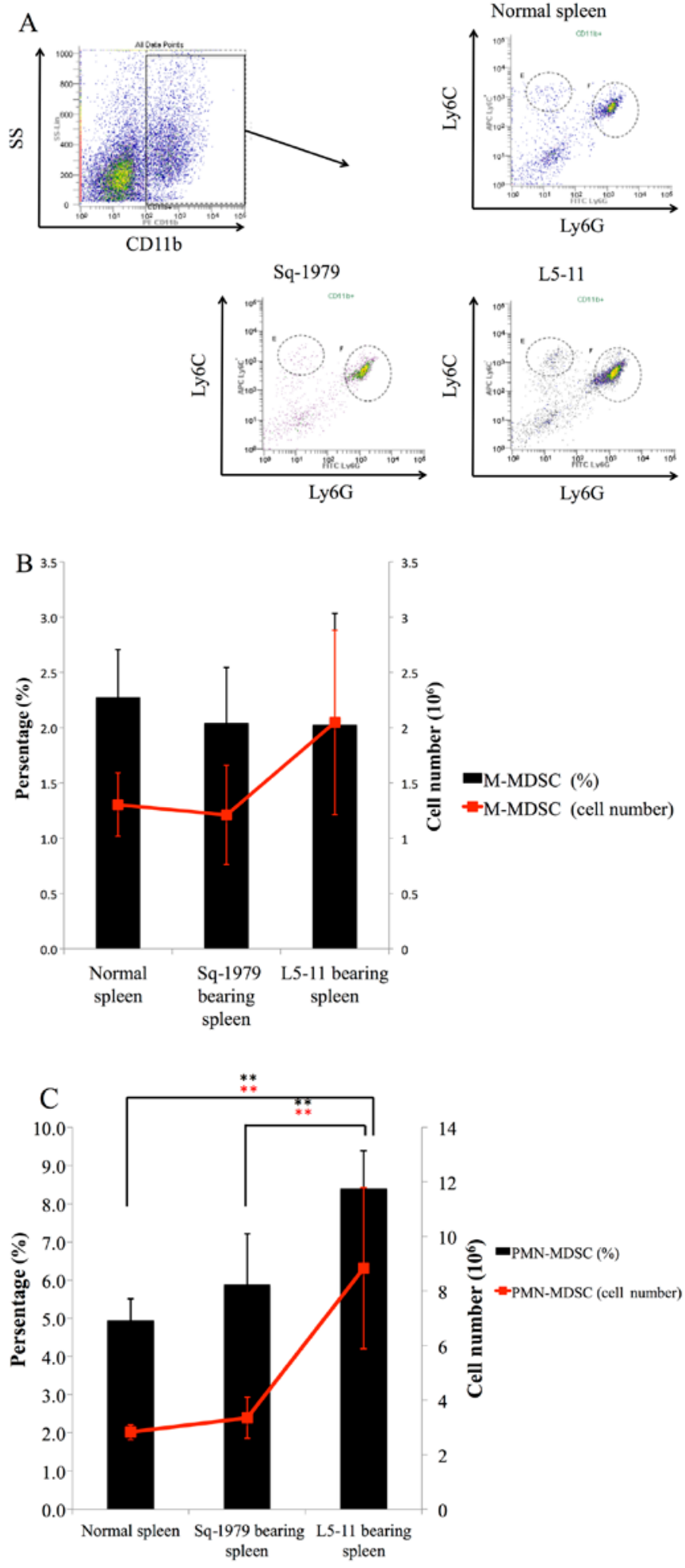
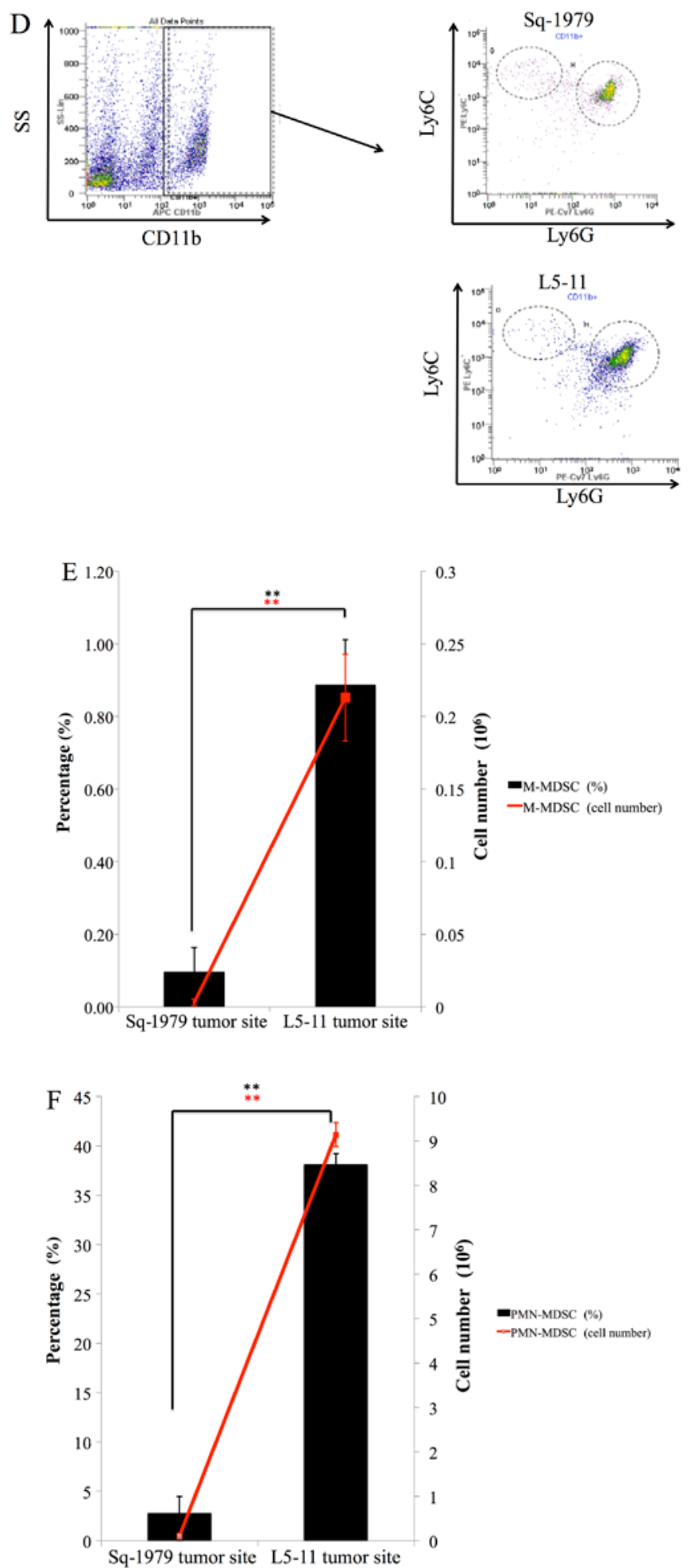

Figure 3. Characterization of MDSCs in the spleen and tumor. (A) Representative single parameter diagrams showing the expression of Ly6C and Ly6G by flow cytometry, gated on CD11 ${ }^{+}$splenic cells. The vertical axis shows Ly6C (APC), and the x-axis shows Ly6G (FITC). (B) Bar graph showing the percentage of M-MDSCs and (C) PMN-MDSCs detected by flow cytometry in the spleen at 21 days after Sq-1979 or L5-11 transplantation. The line graph shows cell counts. (D-F) Graphs are the same as in A-C, except cells were harvested from syngeneic graft tumor tissues, therefore, no samples could be taken from control animals. All quantitative data are represented as mean \pm SD of three independent experiments. ${ }^{*} \mathrm{P}<0.05,{ }^{* *} \mathrm{P}<0.01(\mathrm{n}=12)$.

OSCC cell line Sq-1979, we compared the morphology of the two lines. Sq-1979 cells grew in island structures, similar to the original squamous epithelium. However, L5-11 cells formed fewer of these structures, and when they were observed they were small punctate arrangements that had poor epithelial characteristics (Fig. 1A). Next, we compared the growth rate of these cells. The in vitro growth rate was not significantly different between Sq-1979 and L5-11 cells (Fig. 1B). To test in vivo growth rates, we generated isogenic heterotopic transplantation models from both tumor cell lines and measured 

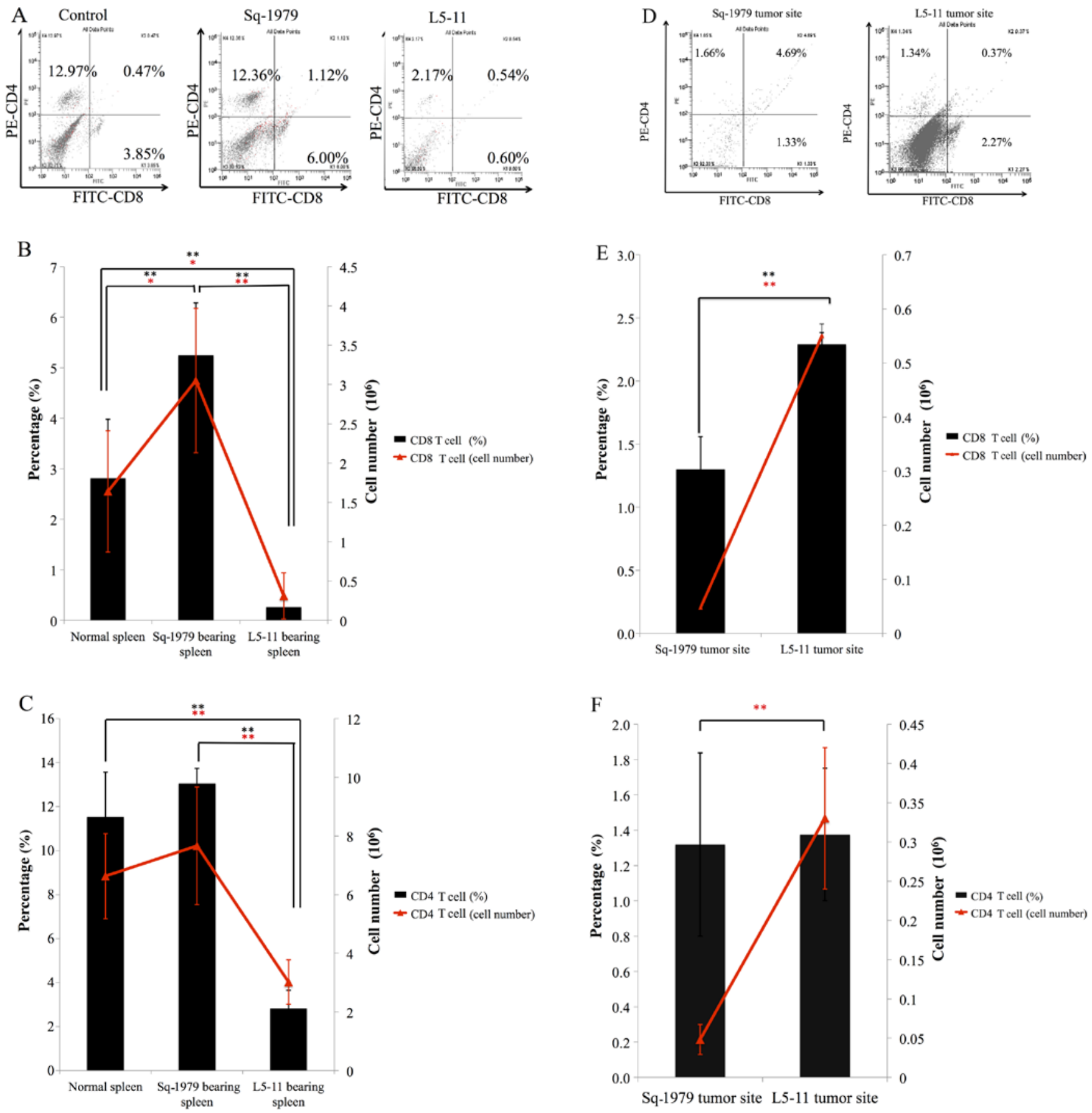

Figure 4. $\mathrm{CD}^{+}$and $\mathrm{CD} 8^{+}$cell from spleen and tumor tissues. (A) Representative single parameter diagrams showing the expression of $\mathrm{CD} 4^{+}$and $\mathrm{CD} 8^{+}$in splenic cells from negative control mice (left panel), Sq-1979-transplanted mice (middle panel) and L5-11-transplanted mice (right panel) by flow cytometry. The vertical axis shows $\mathrm{CD} 4^{+}$(FITC), and the $\mathrm{x}$-axis shows $\mathrm{CD} 8^{+}(\mathrm{PE})$. (B and C) Bar graph from FACS analysis of cell surface (B) $\mathrm{CD} 4{ }^{+}$and $(\mathrm{C}) \mathrm{CD} 8^{+}$ expression that represents the mean \pm SD of three independent experiments. The line graph shows a cell count. (D-F) Graphs are the same as in A-C, except cells were harvested from syngeneic graft tumor tissues, therefore, no samples could be taken from control animals. All quantitative data are represented as mean $\pm \mathrm{SD}$ of three independent experiments. ${ }^{*} \mathrm{P}<0.05,{ }^{* * *} \mathrm{P}<0.01(\mathrm{n}=12)$.

tumor diameters. In contrast to the in vitro growth rates, L5-11 tumors grew faster than Sq-1979 tumors (Fig. 2A). We removed spleens and tumors at day 21 post-transplantation and both were larger in the L5-11 group compared with the Sq-1979 group (Fig. 2B-D).

Metastatic OSCC cell syngeneic grafts induce increased splenic PMN-MDSCs. We examined the population of splenic MDSCs to compare tumor immunogenicity between Sq-1979 and L5-11 syngeneic grafts because, in addition to increased tumor growth in the L5-11 group, the recipient mice also showed significantly increased spleen volume (Fig. 2B-D). The proportion of M-MDSCs were $\sim 2 \%$ from all groups, and there were no significant differences observed. The absolute M-MDSC cell numbers for each group were $1.3 \times 10^{6}$ cells in the control mice, $1.2 \times 10^{6}$ cells from Sq-1979-transplanted mice and $2.0 \times 10^{6}$ cells from L5-11-transplanted mice; these results did not show significant differences (Fig. 3A and B). 

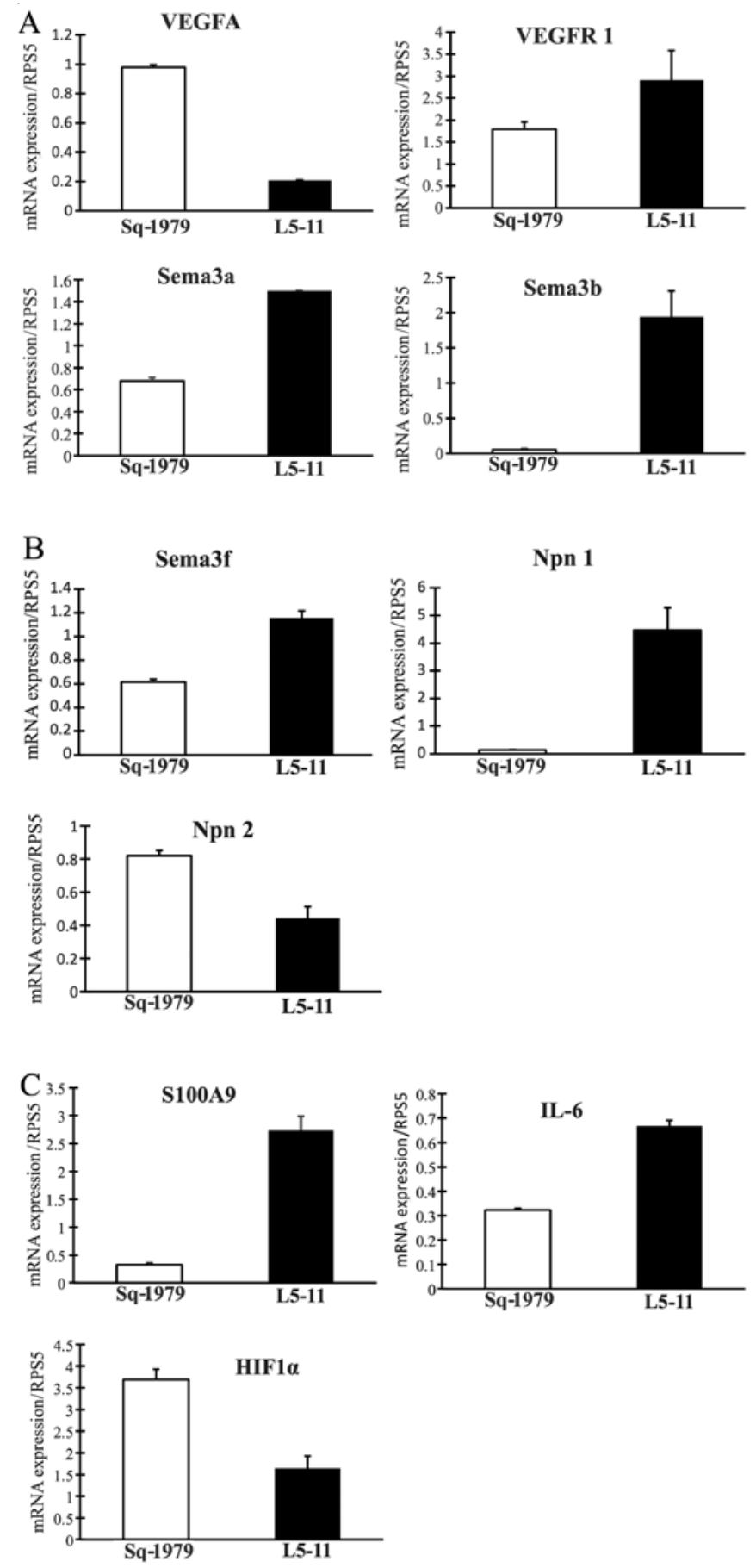

Figure 5. Quantitative real-time PCR analysis of genes that influence MDSC expansion and tumor growth. Quantification of (A) VEGFA, VEGFR1, Sema3a, Sema3b, (B) Sema3f, Npn1, Npn2, (C) S100A9, IL-6 and HIF1a mRNA in Sq-1979 and L5-11 cells using quantitative real-time RT-PCR. The bar graphs show relative expression levels and represent the mean $\pm \mathrm{SD}$ of three independent experiments.

Conversely, the proportion of PMN-MDSCs from L5-11transplanted mice was significantly increased (8.4\%) compared with negative control mice (5.0\%) and Sq-1979-transplanted mice $(5.9 \%)$. These changes were also reflected in the absolute cell numbers of PMN-MDSCs, which were also significantly increased in L5-11-transplanted mice compared with control or Sq-1979-transplanted mice $\left(8.8 \times 10^{6}, 2.8 \times 10^{6}\right.$ and $3.4 \times 10^{6}$ cells, respectively; Fig. $3 \mathrm{~A}$ and $\mathrm{C})$.
Metastatic OSCC cell syngeneic grafts induce increased intratumoral $M$ - and PMN-MDSCs. We concurrently examined the population of intratumoral MDSCs. The absolute cell number of both M-MDSCs and PMN-MDSCs in the tumor were significantly increased in L5-11 compared with Sq-1979 tumors (Fig. 3D-F). Although the fraction of M-MDSCs in L5-11 syngeneic grafts was increased to $0.89 \%$ compared with $0.09 \%$ in Sq-1979, the fraction of PMN-MDSCs from L5-11 markedly increased to $38.1 \%$ compared with $2.7 \%$ in Sq-1979 (Fig. 3D-F). Furthermore, we examined whether the fraction of $\mathrm{CD}^{+}$or $\mathrm{CD}^{+}$cells changed. Splenic $\mathrm{CD}^{+}$and $\mathrm{CD}^{+}$ fractions and cell numbers from L5-11-transplanted mice were lower than the control and Sq-1979-transplanted mice (Fig. 4A-C). Most significantly, the fraction of splenic CD8 ${ }^{+}$ cells from Sq-1979-transplanted mice significantly increased to $5.2 \%$ compared with $2.8 \%$ in the control and $0.3 \%$ in L5-11 (Fig. 4B). The fraction and number of intratumoral CD8 ${ }^{+}$ cells in L5-11 significantly increased compared with Sq-1979 (Fig. 4D and E). Although the number of intratumoral CD4 ${ }^{+}$ cells in L5-11 significantly increased to $0.3 \times 10^{6}$ cells compared with $0.05 \times 10^{6}$ cells in Sq-1979, there was no significant difference in the fraction of intratumoral $\mathrm{CD}^{+}$(Fig. $4 \mathrm{~F}$ ).

Analysis of tumor-derived factors that induce MDSCs. We next explored which factors from tumor cells induce MDSCs. Because the metastasis OSCC cells induced more PMN-MDSCs than the primary OSCC cells in the spleen and tumor (Fig. 3), we hypothesized that tumor-derived factors may be related to the induction of MDSCs. Using real-time PCR to assess the expression of several genes related to MDSCs, we found that VEGFR1, Sema3a, Sema3b, Sema3f, IL-6 and S100A9 levels were significantly increased in L5-11 cells compared with primary Sq-1979 cells (Fig. 5). Conversely, VEGFA, Npn2 and HIF1 $\alpha$ levels decreased despite previous reports suggesting these are regulation factors for MDSCs.

\section{Discussion}

In the present study, we first examined tumor immunogenicity in an isogenic heterotopic tumor transplantation model of a primary OSCC cell line (Sq-1979) and an isogenic heterotopic tumor transplantation model of a lymph node metastasis cell line derived from the Sq-1979 cells. Our data showed that the M-MDSC fraction of the tumor and PMN-MDSC number and fractions of the spleen and tumor in the lymph node metastasis model were significantly increased (Fig. 3). These data indicated that the lymph node metastasis OSCC cells developed the ability to induce PMN-MDSCs in both the spleen and tumor. Therefore, the tumor immune suppression by PMN-MDSCs should be considered a poor prognostic factor for metastasis. Some reports have demonstrated that the primary tumor can induce MDSCs in the spleen and tumor $(14,15)$. The ability of MDSCs to support tumor growth and metastasis can be divided into functions: i) protection of tumor cells from immune-mediated killing; ii) remodeling the tumor microenvironment; iii) establishment of a pre-metastatic niche; and iv) interaction with tumor cells to induce 'stemness' and facilitate epithelial to mesenchymal transition (12). PMN-MDSCs have a similar phenotype as neutrophils and are the largest population of MDSCs, making up $>75-80 \%$ 
of the total MDSCs in tumor-bearing mice (16). This study suggested that both splenic and intratumoral PMN-MDSCs increased not only in the primary tumor model but also in the lymph node metastasis model of OSCC. Moreover, the number and fraction of PMN-MDSCs significantly increased in both the spleen and tumor of the metastasis model compared with the primary tumor model (Fig. 3). Notably, the fraction of intratumoral PMN-MDSCs in the lymph node metastasis model markedly increased to $38.1 \%$ compared with $2.7 \%$ in the primary tumor model (Fig. 3F). Other reports have demonstrated that M-MDSCs possess the main tumor immunosuppressive functions of MDSCs $(17,18)$, suggesting that the immunosuppressive functions of PMN-MDSCs are less robust than M-MDSCs (19). Nevertheless, PMN-MDSCs play a critical role in tumor immunity $(12,20)$, for example, PMN-MDSCs can inhibit antigen-nonspecific T cell immune responses. The proportion of splenic PMN-MDSCs significantly increased to $8.4 \%$ in L5-11-transplanted mice compared with 5\% in controls or 5.9\% in Sq-1979-transplanted mice (Fig. 3C), while, there were no significant differences in the percentage of splenic PMN-MDSC between controls and Sq-1979-transplanted mice (Fig. 3C). Conversely, splenic $\mathrm{CD}^{+}$cells from Sq-1979-transplanted mice significantly increased to $5.2 \%$ compared with $2.8 \%$ in controls; however, in L5-11-transplanted mice there was a significant decrease to $0.3 \%$ compared with controls. These data suggested that $\mathrm{CD} 8^{+}$ cells had been induced in hematopoietic organs of the OSCC primary model, but had been inhibited in the OSCC metastasis model. At first, we anticipated that both the number and fraction of intratumoral $\mathrm{CD}^{+}$and $\mathrm{CD} 8^{+}$cells would be decreased in the metastasis model as in the spleen. However, contrary to our expectations, both the absolute cell numbers and the fraction of intratumoral $\mathrm{CD}^{+}$cells in the metastasis model significantly increased, and the number of intratumoral CD4 ${ }^{+}$ cells significantly increased but the fraction was unchanged (Fig. 4D and F). The augmentation of intratumoral $\mathrm{CD} 4^{+}$cells in the metastasis model may be due to increased numbers of total intratumoral cells compared with the primary tumor model. Although, the fraction of intratumoral $\mathrm{CD} 8^{+}$cells in the metastasis model significantly increased to $2.3 \%$ compared with $1.3 \%$ in the primary model, the augmentation of $\mathrm{CD} 8^{+}$ cells may not be as effective for tumor growth suppression because intratumoral PMN-MDSC in the metastasis model, which have immune suppressive activity against antigenspecific $\mathrm{T}$ cells, increased markedly.

It is often thought that differences in the properties of cancer cells themselves affect the rate of primary tumor growth and metastasis. However, our analysis of syngeneic graft models suggested that the ability of the tumor to induce specific immune cell infiltration may be more important than simple proliferation rates. Therefore, we then analyzed potential tumor-derived factors that may be related to the induction of MDSCs. VEGF is an angiogenic factor secreted from tumor cells, and VEGF signaling through VEGFR1 and Npn1 has been shown to contribute to tumor growth and invasion (21). VEGF production is promoted by HIF-1 $\alpha(22,23)$, and VEGF, IL-6 and S100A9 have been shown to induce the expansion of MDSCs $(24,25)$. The semaphorin family of secreted and membrane-bound ligands that signal through Npn1, 2 enhance angiogenesis and lymphangiogenesis and attract tumor-associated macrophages to the tumor microenvironment (26-28). Therefore, we analyzed the expression of these genes in Sq-1979 and L5-11 cells. The results showed that sema3a, sema3b, sema3f, VEGFR1, Npn1 and S100A9 mRNA levels were increased in L5-11 cells compared with Sq-1979 cells (Fig. 5). These data indicate that acquired expression of these factors in the L5-11 cell line promote tumor growth and metastasis through the construction of pro-tumor microenvironment, inducing angiogenesis and/or inducing the expansion of MDSCs.

In conclusion, our results clearly demonstrate that splenic and intratumoral MDSCs are enhanced in the lymph node metastasis model of OSCC. When assaying for changes in gene expression that could account for the increased expansion of MDSCs, neither VEGF nor HIF-1 $\alpha$ were significantly changed, but VEGFR, S100A9 and IL-6 were significantly enhanced in the lymph node metastasis OSCC cells. These factors may affect the induction of MDSCs, which promote tumor growth and metastasis.

\section{Acknowledgements}

The present study was supported in part by JSPS KAKENHI (grant nos. 24791982, 26462854 and 26861748).

\section{References}

1. Kalnins IK, Leonard AG, Sako K, Razack MS and Shedd DP: Correlation between prognosis and degree of lymph node involvement in carcinoma of the oral cavity. Am J Surg 134: 450-454, 1977.

2. Grandi C, Alloisio M, Moglia D, Podrecca S, Sala L, Salvatori P and Molinari R: Prognostic significance of lymphatic spread in head and neck carcinomas: Therapeutic implications. Head Neck Surg 8: 67-73, 1985.

3. Yu CC, Chen PN, Peng CY, Yu CH and Chou MY: Suppression of miR-204 enables oral squamous cell carcinomas to promote cancer stemness, EMT traits, and lymph node metastasis. Oncotarget 7: 20180-20192, 2016.

4. Ohnishi K, Yamaguchi M, Erdenebaatar C, Saito F, Tashiro H, Katabuchi H, Takeya M and Komohara Y: Prognostic significance of CD169-positive lymph node sinus macrophages in patients with endometrial carcinoma. Cancer Sci 107: 846-852, 2016.

5. Jung EJ, Santarpia L, Kim J, Esteva FJ, Moretti E, Buzdar AU, Di Leo A, Le XF, Bast RC Jr, Park ST, et al: Plasma microRNA 210 levels correlate with sensitivity to trastuzumab and tumor presence in breast cancer patients. Cancer 118: 2603-2614, 2012.

6. Condamine T, Ramachandran I, Youn JI and Gabrilovich DI: Regulation of tumor metastasis by myeloid-derived suppressor cells. Annu Rev Med 66: 97-110, 2015.

7. Heim CE, Vidlak D, Scherr TD, Kozel JA, Holzapfel M, Muirhead DE and Kielian T: Myeloid-derived suppressor cells contribute to Staphylococcus aureus orthopedic biofilm infection. J Immunol 192: 3778-3792, 2014.

8. Gabrilovich DI and Nagaraj S: Myeloid-derived suppressor cells as regulators of the immune system. Nat Rev Immunol 9: 162-174, 2009.

9. Youn JI, Kumar V, Collazo M, Nefedova Y, Condamine T, Cheng P, Villagra A, Antonia S, McCaffrey JC, Fishman M, et al: Epigenetic silencing of retinoblastoma gene regulates pathologic differentiation of myeloid cells in cancer. Nat Immunol 14: 211-220, 2013.

10. Nagaraj S, Schrum AG, Cho HI, Celis E and Gabrilovich DI: Mechanism of $\mathrm{T}$ cell tolerance induced by myeloid-derived suppressor cells. J Immunol 184: 3106-3116, 2010.

11. Weiss JM, Subleski JJ, Back T, Chen X, Watkins SK, Yagita H, Sayers TJ, Murphy WJ and Wiltrout RH: Regulatory T cells and myeloid-derived suppressor cells in the tumor microenvironment undergo Fas-dependent cell death during IL-2/alphaCD40 therapy. J Immunol 192: 5821-5829, 2014. 
12. Marvel D and Gabrilovich DI: Myeloid-derived suppressor cells in the tumor microenvironment: Expect the unexpected. J Clin Invest 125: 3356-3364, 2015.

13. Corzo CA, Condamine T, Lu L, Cotter MJ, Youn JI, Cheng P, Cho HI, Celis E, Quiceno DG, Padhya T, et al: HIF-1 $\alpha$ regulates function and differentiation of myeloid-derived suppressor cells in the tumor microenvironment. J Exp Med 207: 2439-2453, 2010.

14. Khaled YS, Ammori BJ and Elkord E: Increased levels of granulocytic myeloid-derived suppressor cells in peripheral blood and tumour tissue of pancreatic cancer patients. J Immunol Res 2014: 879897, 2014.

15. Walter S, Weinschenk T, Stenzl A, Zdrojowy R, Pluzanska A, Szczylik C, Staehler M, Brugger W, Dietrich PY, Mendrzyk R, et al: Multipeptide immune response to cancer vaccine IMA901 after single-dose cyclophosphamide associates with longer patient survival. Nat Med 18: 1254-1261, 2012.

16. Youn JI, Collazo M, Shalova IN, Biswas SK and Gabrilovich DI: Characterization of the nature of granulocytic myeloid-derived suppressor cells in tumor-bearing mice. J Leukoc Biol 91: 167-181, 2012.

17. Wynn TA: Myeloid-cell differentiation redefined in cancer. Nat Immunol 14: 197-199, 2013

18. Haverkamp JM, Smith AM, Weinlich R, Dillon CP, Qualls JE, Neale G, Koss B, Kim Y, Bronte V, Herold MJ, et al: Myeloidderived suppressor activity is mediated by monocytic lineages maintained by continuous inhibition of extrinsic and intrinsic death pathways. Immunity 41: 947-959, 2014.

19. Bronte V, Brandau S, Chen SH, Colombo MP, Frey AB, Greten TF Mandruzzato S, Murray PJ, Ochoa A, Ostrand-Rosenberg S, et al: Recommendations for myeloid-derived suppressor cell nomenclature and characterization standards. Nat Commun 7: 12150, 2016.

20. Schouppe E, Mommer C, Movahedi K, Laoui D, Morias Y, Gysemans C,Luyckx A, De Baetselier Pand Van Ginderachter JA: Tumor-induced myeloid-derived suppressor cell subsets exert either inhibitory or stimulatory effects on distinct $\mathrm{CD} 8^{+} \mathrm{T}$-cel activation events. Eur J Immunol 43: 2930-2942, 2013.
21. Luo M, Hou L, Li J, Shao S, Huang S, Meng D, Liu L, Feng L, $\mathrm{Xia} \mathrm{P}$, Qin T, et al: VEGF/NRP-1axis promotes progression of breast cancer via enhancement of epithelial-mesenchymal transition and activation of NF- $\kappa \mathrm{B}$ and $\beta$-catenin. Cancer Lett 373 $1-11,2016$.

22. Goel HL and Mercurio AM: VEGF targets the tumour cell. Nat Rev Cancer 13: 871-882, 2013.

23. De Francesco EM, Lappano R, Santolla MF, Marsico S, Caruso A and Maggiolini M: HIF-1 $\alpha /$ GPER signaling mediates the expression of VEGF induced by hypoxia in breast cancer associated fibroblasts (CAFs). Breast Cancer Res 15: R64, 2013.

24. Noman MZ, Desantis G, Janji B, Hasmim M, Karray S, Dessen P, Bronte V and Chouaib S: PD-L1 is a novel direct target of HIF-1 $\alpha$, and its blockade under hypoxia enhanced MDSC-mediated T cell activation. J Exp Med 211: 781-790, 2014.

25. Chen X, Eksioglu EA, Zhou J, Zhang L, Djeu J, Fortenbery N, Epling-Burnette P, Van Bijnen S, Dolstra H, Cannon J, et al: Induction of myelodysplasia by myeloid-derived suppressor cells. J Clin Invest 123: 4595-4611, 2013.

26. Miao HQ, Lee P, Lin H, Soker S and Klagsbrun M: Neuropilin-1 expression by tumor cells promotes tumor angiogenesis and progression. FASEB J 14: 2532-2539, 2000

27. Casazza A, Laoui D, Wenes M, Rizzolio S, Bassani N, Mambretti M, Deschoemaeker S, Van Ginderachter JA, Tamagnone L and Mazzone M: Impeding macrophage entry into hypoxic tumor areas by Sema3A/Nrp1 signaling blockade inhibits angiogenesis and restores antitumor immunity. Cancer Cell 24: 695-709, 2013.

28. Hu ZQ, Zhou SL, Zhou ZJ, Luo CB, Chen EB, Zhan H, Wang PC, Dai Z, Zhou J, Fan J, et al: Overexpression of semaphorin 3A promotes tumor progression and predicts poor prognosis in hepatocellular carcinoma after curative resection. Oncotarget 7: 51733-51746, 2016. 